\title{
Biomimetic hydrogel-CNT network induced enhancement of fluid-structure interactions for ultrasensitive nanosensors
}

\author{
Meghali Bora ${ }^{1,4}$, Ajay Giri Prakash Kottapalli, ${ }^{1,4}$, Jianmin Miao $^{2}$ and Michael S Triantafyllou ${ }^{3}$
}

Flexible, self-powered, miniaturized, ultrasensitive flow sensors are in high demand for human motion detection, myoelectric prosthesis, biomedical robots, and health-monitoring devices. This paper reports a biomimetic nanoelectromechanical system (NEMS) flow sensor featuring a PVDF nanofiber sensing membrane with a hydrogel infused, vertically aligned carbon nanotube (VACNT) bundle that mechanically interacts with the flow. The hydrogel-VACNT structure mimics the cupula structure in biological flow sensors and gives the NEMS flow sensor ultrahigh sensitivity via a material-induced drag force enhancement mechanism. Through hydrodynamic experimental flow characterization, this work investigates the contributions of the mechanical and structural properties of the hydrogel in offering a sensing performance superior to that of conventional sensors. The ultrahigh sensitivity of the developed sensor enabled the detection of minute flows generated during human motion and micro-droplet propagation. The novel fabrication strategies and combination of materials used in the biomimetic NEMS sensor fabrication may guide the development of several wearable, flexible, and self-powered nanosensors in the future.

NPG Asia Materials (2017) 9, e440; doi:10.1038/am.2017.183; published online 27 October 2017

\section{INTRODUCTION}

Nature's designs have long inspired researchers aiming to find technical solutions and develop novel and advanced engineering systems. ${ }^{1}$ Biological sensors are an example of a natural design that has intrigued scientists over the years. These sensors, for example, flow sensors, acoustic sensors, and chemical sensors, have developed high efficiencies, compactness, responsivity, and sensitivity through evolution over billions of years. ${ }^{2}$ Most man-made artificial sensors scarcely match the performance of these natural sensors. Hence, for the development of novel sensors with high performance and sensitivity, it is desirable to apply designs from natural sensors to artificial engineering materials.

Hair cell mechanoreceptors belong to a class of well-developed natural sensors ubiquitously found in many living organisms such as insects, amphibians, fishes, and mammals. ${ }^{2}$ These natural transducers respond to a variety of environmental stimuli and are capable of sensing pressure, flow, acoustic waves, and chemicals. Some examples include air flow sensors in crickets, air flow and vibration sensors in spiders, water flow sensing whiskers in harbor seals, neuromast acceleration sensors in fishes, and cilia in the organs of mammals, including the taste buds on the tongue and cilia in the nose, lungs, and inner ear cochlea. ${ }^{1-3}$ In aquatic environments, these hair cells have special significance because they form a basic and highly effective mechanism for flow sensing and environmental perception.

Several researchers have developed hair cell-based microelectromechanical system (MEMS) flow sensors that were inspired by the mechanosensory lateral line of fish, by using piezoelectric and piezoresistive membranes with cylindrical, polymeric standing structures. ${ }^{4-7}$ To improve the sensitivity of these artificial MEMS sensors, biomimetic approaches have been investigated to mimic the specialized structures of the neuromast, such as the cupula. In the microfabricated sensors, the cupula and cupular fibrils have been mimicked by using hydrogel capping and electrospun micro/nano fibers, respectively. ${ }^{8-11}$ Such incorporation of biomimetic materials results in an increase in the sensitivity and threshold detection of the hair cell sensors by several orders of magnitude. A soft gelatinous structure, called cupula, covers the sensory organ, that is, the neuromasts, of fishes. There are two types of neuromasts, superficial neuromasts (SNs), which are present on the skin surface and canal neuromasts $(\mathrm{CNs})$, which are present just below the skin inside fluid-filled channels or canals. The neuromasts act as a medium that couples the underlying mechanosensing cells to the outside water flow through viscous forces. ${ }^{12}$ They enhance the drag forces and the absorption of the flow stimulus via short hair bundles and transmission to the sensory hair cells beneath. ${ }^{13}$

${ }^{1}$ Center for Environmental Sensing and Modeling (CENSAM) IRG, Singapore-MIT Alliance for Research and Technology (SMART), Singapore, Singapore; ${ }^{2}$ School of Mechanical \& Aerospace Engineering, Nanyang Technological University, Singapore, Singapore and ${ }^{3}$ Department of Mechanical Engineering, Massachusetts Institute of Technology, Cambridge, MA, USA

${ }^{4}$ These authors contributed equally to this work.

Correspondence: Dr AGP Kottapalli, Center for Environmental Sensing and Modeling (CENSAM) IRG, Singapore-MIT Alliance for Research and Technology (SMART), 1 Create Way, \#09-03, Create Tower, Singapore 138602, Singapore.

E-mail: ajay@smart.mit.edu

Received 6 March 2017; revised 6 August 2017; accepted 18 August 2017 
Besides the increase in the overall cross-sectional area that is exposed to flow, the enhanced drag forces are also a result of factors related to the material properties of the cupula. The biological cupula is composed of $90 \%$ water, and its material composition (and especially the hydrophilicity and permeability characteristics) plays a critical role in signal transmission. ${ }^{13}$ In certain fishes, the cupula also has long thin fibrils that run along the length of the cupula. ${ }^{14}$ These fibrils act as a scaffold that supports the cupula structure, thus allowing it to grow taller and away from the stagnant boundary layer of the fish's skin surface, ${ }^{9}$ thereby increasing the aspect ratio of the overall sensory organs and their sensitivity. These fibrils are also thought to help in coupling the underlying ciliary bundles to the cupula, thus facilitating the mechanotransduction process in transmitting mechanical energy to generate neuroelectrical pulses. ${ }^{13}$

Biological neuromast sensors exhibit complex designs with multifunctional properties, hierarchical structures, and diverse materials. ${ }^{15}$ The fabrication technologies used in developing hair cell inspired MEMS sensors so far, consider designs based on basic structures and do not mimic the morphological features, materials and dimensional variations. ${ }^{8}$ An amalgamation of the state-of-the-art nanofabrication technologies and biomimetic soft materials is required to emulate biological sensors and develop ultrasensitive and self-powered artificial nanosensors. Here we propose a novel biomimetic nanoelectromechanical systems (NEMS) flow sensor that is ultra-sensitive, self-powered, flexible in structure, and easy to fabricate. We achieved this by combining biological design with nanofabrication technology and biomimetic materials. The sensor features an electrospun and aligned polyvinylidene fluoride (PVDF) nanofiber membrane and hyaluronic acid (HA) hydrogel infused vertically aligned carbon nanotube (VACNT) bundle. The use of electrospun PVDF nanofibers as the fundamental sensing elements offers numerous benefits, such as selfpowering, flexibility, high elasticity, high sensitivity (due to the high piezoelectric coefficient), low cost, ease of fabrication, dimensional stability, biocompatibility, chemical inertness, high mechanical resistance and light weight. ${ }^{16-18}$ Flow sensors such as the ones developed in this work are in demand in emerging fields such as electronic skin, human motion detection, myoelectric prosthetic limbs, artificial skin, wearable devices, energy harvesters, and microfluidics.

\section{MATERIALS AND METHODS}

\section{Electrospinning of PVDF}

PVDF powder (MW $534000 \mathrm{Da}$ ), dimethyl formamide (DMF), and acetone were purchased from Sigma Aldrich, Singapore. PVDF (1.5 g) was dissolved in a co-solvent mixture of $3 \mathrm{ml} \mathrm{DMF}$ and $7 \mathrm{ml}$ acetone (in a ratio of 1:2.33) to obtain a $15 \%(\mathrm{w} / \mathrm{v})$ solution. Stirring the solution at $70{ }^{\circ} \mathrm{C}$ for $60 \mathrm{~min}$ and then incubating it in an oven at $70^{\circ} \mathrm{C}$ for another $30 \mathrm{~min}$ homogeneously dissolved the PVDF. The resulting clear and viscous PVDF solution was filled in a $10 \mathrm{ml}$ syringe fixed with an $18 \mathrm{G}$ needle for electrospinning. The PVDF solution syringe was fitted onto a pump, and the flow rate was set to $5 \mu \mathrm{min}^{-1}$. The rotating spindle that collects the nanofibers was positioned at a distance of $15 \mathrm{~cm}$ from the needle, and a direct current (DC) voltage of $12 \mathrm{kV}$ was applied across the needle and spindle. The spindle, which was $10 \mathrm{~cm}$ in diameter, was covered with aluminum (Al) foil as the collecting substrate, and the rotating speed was set to 1500 r.p.m. As the polymer solution drop was electrostatically stretched or pulled toward the spindle, the nanofibers formed and laid on the foil in an aligned manner, owing to the continuous rotation.

\section{Synthesis of the HA hydrogel cupula}

The modification of hyaluronic acid (HA) using tyramine (Tyr), and crosslinking tyramine modified HA (HA-Tyr) with hydrogen peroxide $\left(\mathrm{H}_{2} \mathrm{O}_{2}\right)$ in the presence of horseradish peroxidase (HRP) via covalent bond formation is a well-established method. ${ }^{19}$ Corgel hydrogels (Corgel BioHydrogel Lifecore kit,
Lifecore Biomedical, Chaska, USA) are commercially available HA hydrogels based on this modification and crosslinking mechanism. The Corgel polymer kit purchased for this work included HA-Tyr with a 5.5\% degree of modification, $10 \mathrm{U} \mathrm{ml}^{-1} \mathrm{HRP}$, and $1 \% \mathrm{H}_{2} \mathrm{O}_{2}$ in phosphate buffer saline (PBS). The Corgel 5.5 hydrogels were prepared according to the instructions provided by the supplier (Lifecore Biomedical). HA-Tyr powder was dissolved in HRP (in PBS) to obtain a $1.2 \%(\mathrm{w} / \mathrm{v})$ solution. The original stock solution of $\mathrm{H}_{2} \mathrm{O}_{2}$ was diluted with water to obtain a $0.3 \%$ concentration. After overnight stirring, the HA-Tyr and HRP polymer solution was mixed with $\mathrm{H}_{2} \mathrm{O}_{2}$ in the ratio of 1:25 (v/v) $\left(\mathrm{H}_{2} \mathrm{O}_{2}: \mathrm{HA}-\mathrm{Tyr}\right)$ to initiate gelation. Gelation began immediately, and the crosslinking reaction was allowed to continue overnight. The next day the hydrogels were immersed in deionized (DI) water and allowed to swell for $24 \mathrm{~h}$ at room temperature (RT).

\section{Characterization of hydrogels}

The water-swollen HA-Tyr hydrogels were characterized on the basis of their morphology, network structure parameters, and mechanical behavior.

\section{Morphology}

The morphology of the HA-Tyr hydrogels was observed using scanning electron microscopy (SEM) (JSM 6360A Jeol, Japan). Water-swollen hydrogel samples were rapidly quenched in liquid nitrogen for few seconds, then lyophilized for 5 days. The freeze-dried hydrogels were gold coated for $80 \mathrm{~s}$, and their surface and cross-sectional areas were examined under SEM.

\section{Swelling}

The network structure parameters of the HA-Tyr hydrogels were calculated using the Flory-Rehner equations, as previously described. ${ }^{20}$ The HA-Tyr hydrogels were swollen for $24 \mathrm{~h}$ in DI water, and their wet mass was recorded. The gels were then lyophilized for 5 days, and their dry mass was recorded. The swelling ratio, with respect to mass, was used to calculate other parameters such as the volume swelling ratio, molecular weight between crosslinks, crosslinking density, and mesh size.

\section{Rheology}

The mechanical properties of HA-Tyr hydrogels were evaluated using a stresscontrolled rheometer (Physica MCR 501, Anton Parr, USA) and a parallel plate geometry measuring system (PP25/TGSN 6539, diameter $25 \mathrm{~mm}$ ). All the experiments were performed at a RT of approximately $23^{\circ} \mathrm{C}$. The waterswollen hydrogels were subjected to dynamic oscillation shear tests with either a constant frequency or amplitude. In the amplitude sweep, the storage modulus $\left(G^{\prime}\right)$, and loss modulus $\left(G^{\prime \prime}\right)$ were obtained as a function of the strain rate varying from 0.005 to $5 \%$ at $1.6 \mathrm{~Hz}$. In the frequency sweep test, $\mathrm{G}^{\prime}, \mathrm{G}^{\prime \prime}$, and complex viscosity were obtained as a function of the frequency varying from 0.1 to $10 \mathrm{~Hz}$ at a $0.05 \%$ strain rate. This strain was selected from the linear viscoelastic (LVE) region observed in the amplitude sweep plot.

\section{Morphological characterization of the CNT pillar with the HA-Tyr hydrogel}

The HA-Tyr hydrogel infused CNTs were characterized for their morphology using field emission SEM (FESEM) (JSM 6701F, Jeol Asia Pte Ltd, Singapore). The samples with CNTs and hydrogel were freeze dried for 5 days, coated with platinum for $80 \mathrm{~s}$, and examined through FESEM.

\section{Flow sensing experiments}

A dipole (sphere of $16 \mathrm{~mm}$ diameter) stimulus was used in all the experiments to generate oscillatory flows with various velocities. The dipole was vibrated using a permanent magnet mini-shaker (Brüel \& Kjær model 4810, Norcross, GA; axial resonant frequency $>18 \mathrm{kHz}$ ) and was connected using a rigid stainless-steel rod $4 \mathrm{~mm}$ in diameter. The mini-shaker was inverted and mounted so that the dipole was positioned $25 \mathrm{~mm}$ away from the sensor and vibrated perpendicular to the long axis of the VACNT bundle. The velocity generated by the vibration of the dipole was pre-calibrated using a Polytec PSV-300 laser Doppler vibrometer (LDV). ${ }^{7}$ For all the experimental results, the unamplified peak-to-peak output of the sensor was plotted. The voltage output 
from the sensor was acquired using a National Instruments data acquisition card (NI-DAQ) and recorded using the LabVIEW software.

\section{RESULTS AND DISCUSSION}

\section{Development of the biomimetic NEMS neuromast sensor}

Sensor structure and sensing principle. The sensor consisted of three main parts: the PVDF nanofiber sensing membrane, a high aspect ratio VACNT bundle, and a hydrogel cupula. The PVDF membrane is a self-suspended film of aligned PVDF nanofibers fabricated through electrospinning process. The circular membrane has a thickness of $\sim 20 \mu \mathrm{m}$, defined by the timed electrospinning, and a diameter of $2 \mathrm{~mm}$, defined by a cavity formed in the two optically clear adhesive (OCA) films between which the membrane is suspended.

The sensor features a cylindrical hair-like structure formed from highly aligned vertically standing CNT bundles (CVD Equipment Corp, New York, NY, USA). A single VACNT bundle is $4000 \mu \mathrm{m}$ in height and $350 \mu \mathrm{m}$ in diameter. The high aspect ratio VACNT (11.43) offers a high sensitivity to the sensor as compared to those with shorter hair structures that fall within the flow generated boundary layers and thereby experience a reduced velocity. ${ }^{5,21,22}$ The VACNT bundle, which is positioned at the center of the circular membrane is attached at its root but free to move at its distal tip, and is encapsulated within a HA-based hydrogel canopy mimicking the cupula of biological neuromast. A prolate spheroid-shaped hydrogel cupula with the same height as the CNT bundle and a diameter 4.3 times that of the bundle encapsulates three-quarters of the bundle from its distal end.

Flows generated in the vicinity of the sensor would cause a drag force on the hydrogel cupula, owing to the flow-structure interaction. The cupula transduces the flow-induced drag force into a displacement in the VACNT bundle. Since the VACNT bundle is fixed to the PVDF nanofiber membrane on one end, the bending moment in the bundle causes a displacement in the membrane. Due to the piezoelectric nature of the PVDF nanofibers, electrical charges are generated, which are acquired as the sensor output through the copper contacts. In response to the flow, the rigid nature of the CNT bundle causes it to pivot at the base, rather than bend along the shaft, thus maximizing the tip displacement and torque at the base, increasing the strain induced in the PVDF fibers, which in turn leads to enhanced sensitivity of the sensor.

Sensor fabrication. The sensor fabrication comprises three main parts: (1) electrospinning to form the self-suspended PVDF sensing membrane; (2) atmospheric pressure chemical vapor deposition (APCVD) growth of the multi-walled VACNT bundles; and (3) artificial cupula synthesis and drop-casting. Figures la-f depict the sensor fabrication through schematics. More details regarding the unit processing steps involved in the sensor fabrication are provided in the Supplementary Information (Supplementary Figure S1). The electrospinning parameters were optimized to obtain PVDF nanofibers with a high $\beta$-phase. The electrical and mechanical properties of the PVDF nanofibers and the porosity of the VACNT bundle were characterized to evaluate their effects on the sensitivity and accuracy of the sensor. The piezoelectric coefficient of a single nanofiber, which was determined through fiber displacement in the presence of a varying electric field, was $-58.7 \mathrm{pm} \mathrm{V}^{-1}$. Nanomechanical characterizations conducted using a triboindenter revealed a Young's modulus and hardness of 2.2 and $0.1 \mathrm{GPa}$, respectively. The VACNT bundle was characterized for its porosity using Brauner-Emmett-Teller (BET) analysis, and the average pore diameter was $74.12 \AA$. a

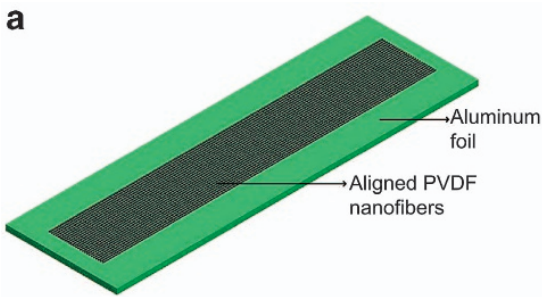

d
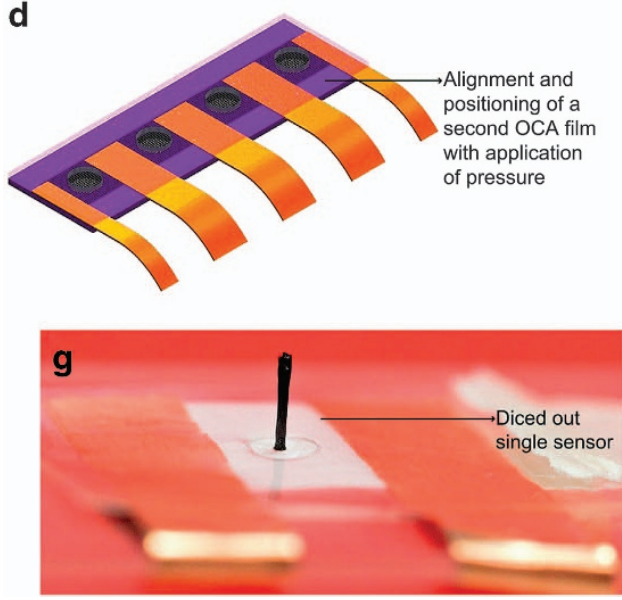

b

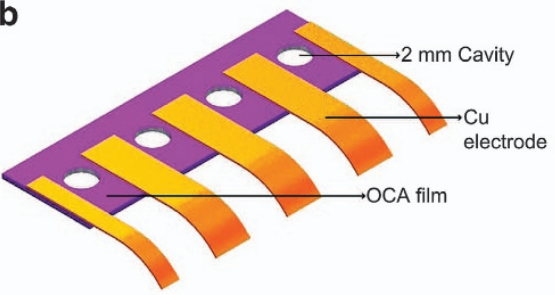

e

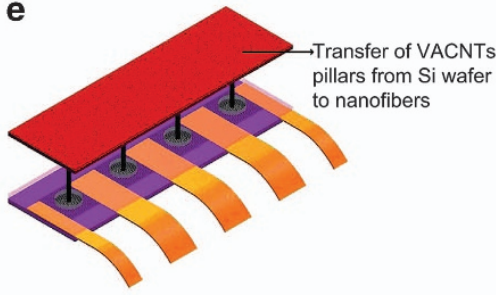

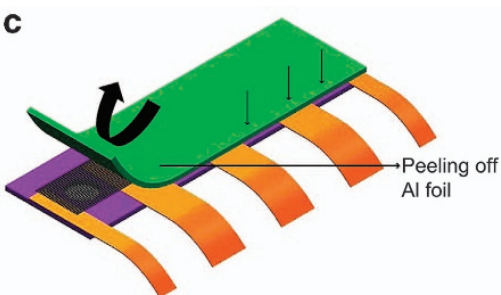

C

f

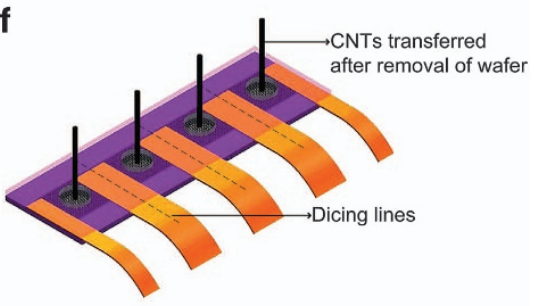

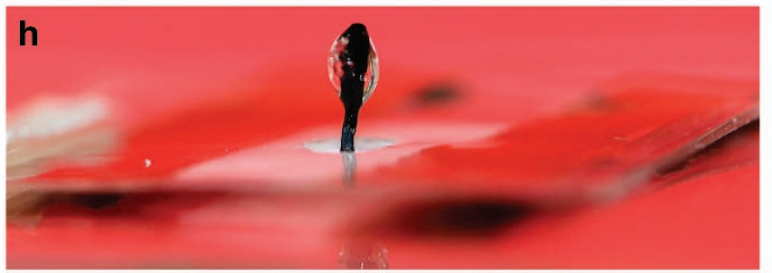

Figure 1 Fabrication of the biomimetic NEMS sensor. (a) Aligned PVDF nanofibers electrospun on top of aluminum foil. (b) OCA film punched with cavities $2 \mathrm{~mm}$ in diameter. (c) Nanofibers transferred from the aluminum foil to the OCA film. (d) Another identical OCA film was placed, and the fibers were sealed between the two OCA films via application of pressure. (e) The CNT bundles separately grown on a silicon wafer were batch-transferred to the array of PVDF films. (f) After the CNT transfer process, the silicon wafer was removed. (g) Photograph of a single sensor featuring the vertically standing VACNT at the center of the PVDF membrane. (h) Photograph of the sensor after drop-casting the HA-Tyr hydrogel on the VACNT bundle. 
Hydrogel cupula. For the artificial cupula, the HA-based hydrogel was used and drop cast over the VACNT bundle. A modified HA was used to develop the chemically crosslinked hydrogel. Commercially available HA-Tyr (Corgel), was enzymatically crosslinked using a wellestablished method that results in the formation of a hydrogel with controlled properties. ${ }^{19,23}$ The HA-Tyr polymer chains were covalently bound by using hydrogen peroxide $\left(\mathrm{H}_{2} \mathrm{O}_{2}\right)$ in the presence of horseradish peroxidase (HRP) enzyme. Figures $1 \mathrm{~g}$ and $\mathrm{h}$ show the naked VACNT bundle sensor and the sensor with the HA-Tyr hydrogel cupula, respectively.

HA-Tyr hydrogel, used to encapsulate the CNT bundle, mimics the material, structural, and mechanical properties of the cupula of CNs in fishes with a rigid yet highly porous network structure. HA is a glycosaminoglycan, thus mimicking the material composition of natural cupula. ${ }^{14}$ The hydrogel synthesis was optimized to obtain a high mechanical strength (Young's modulus similar to that of the cupula of $\mathrm{CNs}$ ) and water content inside its matrix mimicking the natural properties of the cupula. After the HA-Tyr was completely crosslinked, it was immersed in DI water to achieve a swelling equilibrium. Figure 2a shows a photograph of the HA-Tyr hydrogel disc after swelling. The hydrogel was very transparent with a smooth surface. To observe its mesh network, this water swollen gel was lyophilized and examined through SEM. Figure $2 \mathrm{~b}$ shows a scanning electron micrograph of the cross-section of a dried hydrogel sample. The gel showed a porous network capable of absorbing and retaining water molecules in its matrix. This result was further corroborated by the results of the swelling study. The network structure parameters, including the swelling ratio and mesh size, were calculated by using the Flory Rehner equations for polymers, as described previously. ${ }^{20}$ Supplementary Table S1 (see Supplementary Information) summarizes the approximate values of each of these parameters. The water content of these gels was $\sim 99 \%$ with micrometer-sized pores in their network. Thus, this biomimetic cupula possesses a high hydrophilicity and a porous structure that would affect the frictional forces resulting from the viscous drag of fluid motion. Figures $2 \mathrm{c}-\mathrm{e}$ show the plots obtained from the rheological analysis of the HA-Tyr hydrogel for its mechanical properties. In the amplitude sweep test, the $\mathrm{G}^{\prime}$ was plotted as a function of the strain rate, as shown in Figure 2c. From the LVE region of this plot, a strain rate was selected, and frequency sweeps in Figures 2d and e were obtained with the storage modulus and complex viscosity, respectively, plotted as a function of the frequency. The modulus was constant with respect to the frequency up to approximately $5 \mathrm{~Hz}$, thus demonstrating the high stiffness of these gels. Additionally, the complex viscosity consistently decreased with an increase in the frequency, thus showing the shear thinning behavior of the HA-Tyr hydrogels, which is a characteristic of viscoelastic polymers. The storage modulus at $1 \mathrm{~Hz}(\sim 3.46 \mathrm{kPa})$ was used to calculate the Young's modulus (E) using the relation $E=3 \times \mathrm{G}^{\prime}{ }^{9}$ The HA-Tyr hydrogels had $\mathrm{E}$ value of approximately $10.4 \mathrm{kPa}$, which is similar to those for the biological cupula of CNs. ${ }^{10}$ The high stiffness and water content of the HA-Tyr hydrogel network demonstrates a rigid, yet, highly porous structure. This is typical of the cupula of CNs, making the HA-Tyr hydrogels a good mimic of the natural cupula.

VACNT bundle. Figure 3a shows a photograph of the high aspect ratio (11.43) CNT bundles grown on a silicon wafer. Figures $3 \mathrm{~b}$ and $\mathrm{c}$ are FESEM images of a CNT bundle at different magnifications, showing thousands of nanotubes within a bundle and the high alignment along the length of growth, respectively. CNTs are hydrophobic; thus, to facilitate hydrogel drop casting, they were exposed to oxygen plasma treatment. ${ }^{24}$ For drop-casting, the HA-Tyr solution with HRP was dropped over the plasma-exposed CNTs and then crosslinked by dropwise addition of $\mathrm{H}_{2} \mathrm{O}_{2}$ solution. Gelation was
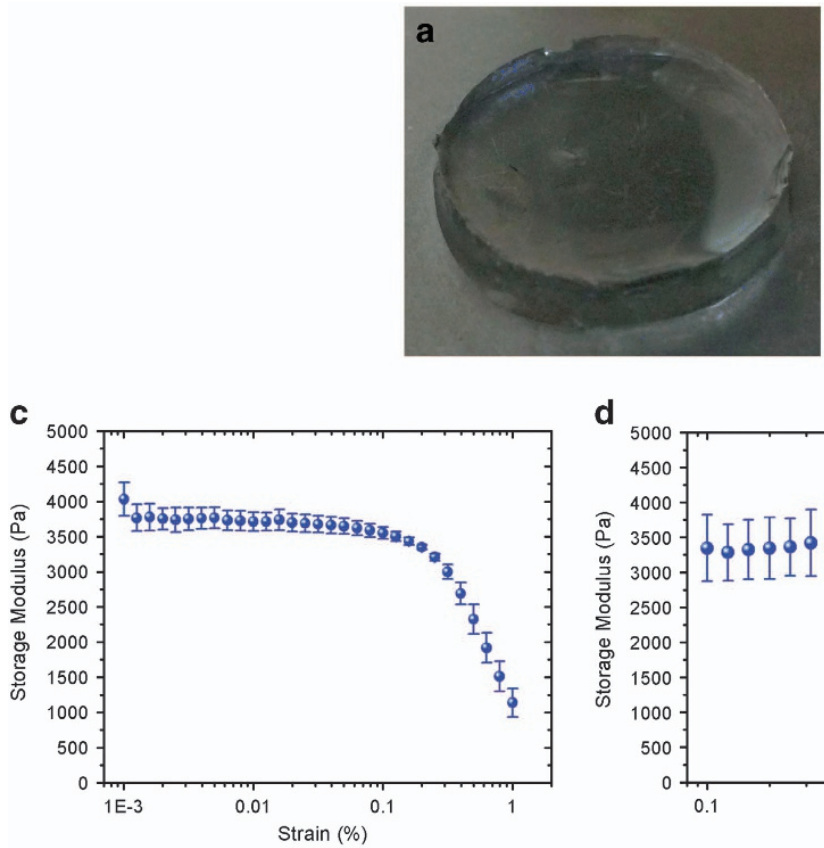

d

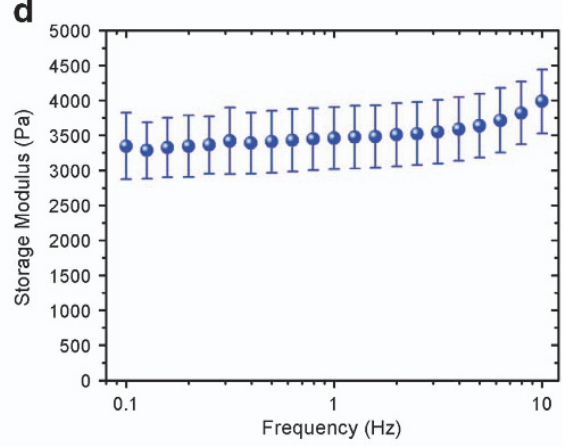

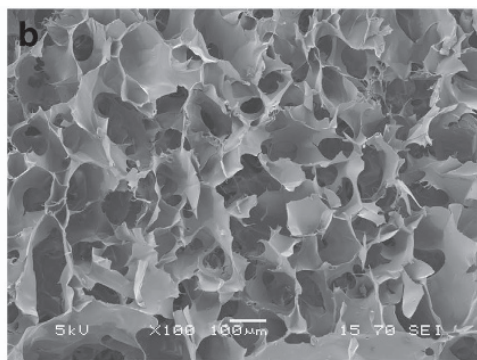

e

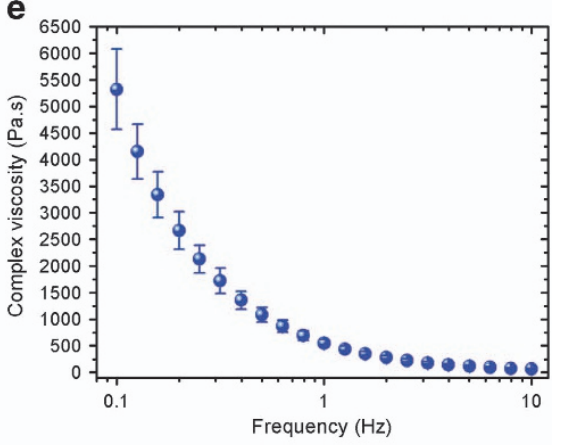

Figure 2 Characterization of the HA-Tyr hydrogels. (a) A photograph of a hydrogel disc after crosslinking and swelling in DI water. (b) Scanning electron micrograph of hydrogel showing the porous mesh network. (c) Storage modulus of hydrogel as a function of strain rate, showing constant stiffness up to $0.1 \%$ strain. (d) Storage modulus of hydrogels as a function of frequency, showing a high stiffness across the entire range tested. (e) Complex viscosity as a function of frequency showing shear thinning behavior. 

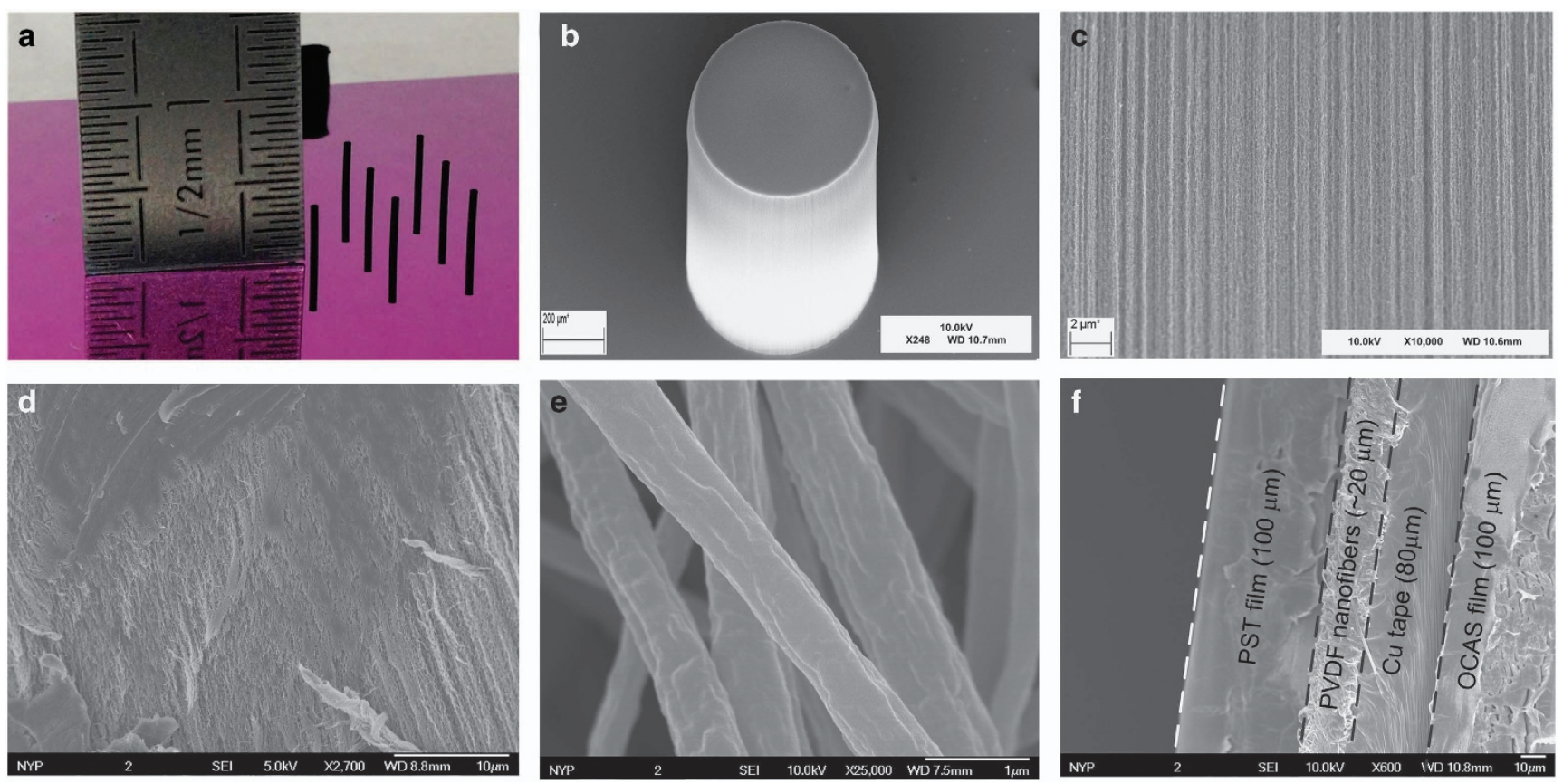

Figure 3 Characterization of the VACNT bundle and PVDF nanofibers. (a) Photograph showing an array of VACNTs as-grown on a Si wafer. The scale placed alongside shows their high aspect ratio. (b) FESEM image showing the angle view of a single CNT bundle on a Si wafer after completion of the growth. (c) FESEM image of a CNT bundle showing the high alignment of the nanotubes running parallel to each other. (d) FESEM image of the cross-section of the hydrogel cupula-dressed CNT bundle showing the infusion of the hydrogel over and in between the nanotubes. (e) FESEM image of the aligned, electrospun PVDF nanofibers. (f) FESEM image of the PVDF nanofiber membrane suspended between two OCA films.

allowed to continue overnight, and the hydrogel was swollen in DI water for $24 \mathrm{~h}$. Figure $3 \mathrm{~d}$ shows a FESEM image of the cross-section of the CNT bundle infused with hydrogel. It can be observed that the hydrogel was present on the CNTs as well as in between them. This is because during swelling, as the water diffused into the hydrogel network and the polymer chains started to swell, they would also open up the CNT bundles by infusing into them due to swelling. This infusion of water-filled polymer chains into CNTs would help them to more efficiently couple the mechanical deflection stimulus to the nanofibers. Figure 3e shows a FESEM image of the aligned PVDF nanofibers (thickness $\sim 700 \mathrm{~nm}$ ), and Figure $3 \mathrm{f}$ shows a cross-section of the PVDF membrane (thickness $\sim 20 \mu \mathrm{m}$ ) that was suspended between the two OCA films.

\section{Sensor testing}

The biomimetic NEMS flow sensor was experimentally characterized in air and water flows to evaluate its flow-sensing performance. To investigate the mechanical and material contributions to the sensitivity enhancement offered by the HA-Tyr hydrogel cupula, a comparative experimental analysis was conducted. This process involved a comparison of the flow-sensing performance of the PVDF membrane sensor featuring just the VACNT bundle and the VACNT bundle scaffolding the hydrogel cupula in both air and water flows. In all the flow-sensing experiments, a dipole (vibrating sphere) stimulus was used to generate flows in the vicinity of the sensors. The choice of a dipole stimulus for the experiments is due to its biological relevance to hydrodynamic flow sensing in CNs in fishes. The flow field generated by an oscillating dipole in water closely represents that generated by a freely swimming fish, which forms the cues for CNs that are majorly responsible for object localization. ${ }^{25,26}$ In addition, the dipole stimulus forms experimental scenarios similar to those used in past studies by biologists and engineers for the characterization of artificial hair cell sensors, which allows comparison of the sensing performance. ${ }^{2-13,21,22,27,28}$ To generate varying flow velocities, the dipole was driven with a sinusoidal signal of fixed amplitude of $250 \mathrm{mV}_{\mathrm{rms}}$ and varying frequencies from $1-100 \mathrm{~Hz}$. When the dipole was set to oscillate at distinct frequencies, the sensor output was verified to follow a sinusoidal trend at the same frequency of the source in both air and water media. The experimental setup used in this work for the oscillatory flow sensing in water is shown in Figure 4a. For air flow sensing, the setup was similar except that the medium was ambient air. The initial flow sensing experiments were conducted using the PVDF sensor with a naked VACNT bundle without the cupula dressing. The dipole was located $25 \mathrm{~mm}$ away from the sensor and vibrated in a plane perpendicular to the long axis of the VACNT bundle. The vibration of the sphere causes the fluid surrounding it to vibrate at the same frequency. The drag force generated by displaced water causes the VACNT bundle to move at the same frequency as that of the dipole.

To characterize the threshold detection limit of the sensor and flow sensitivity, the velocity of the oscillatory flow was varied from 1 to $100 \mathrm{~mm} / \mathrm{s}^{-1}$, and the sensor output was recorded at each velocity. Figures $4 \mathrm{~b}$ and $\mathrm{c}$ show the flow sensing results conducted in air and water, respectively. The sensor output monotonically increased with the increase in flow velocity, as anticipated. Error bars show the s.d.'s of five repeats of the experiments using five of the fabricated sensors. The as-recorded sensor output data are provided in the Supplementary Information for the lowest and highest velocity points (Supplementary Figure S3). Notably, the flow-structure interaction induced drag force depends on the contributions from both the skin friction and pressure gradient (pressure on both sides of the VACNT bundle that drives its motion). At low Reynolds numbers $\left(\mathrm{R}_{\mathrm{e}}<50\right)$, skin friction is the dominant factor contributing to the drag force. At higher values of $R_{e}$, 
a
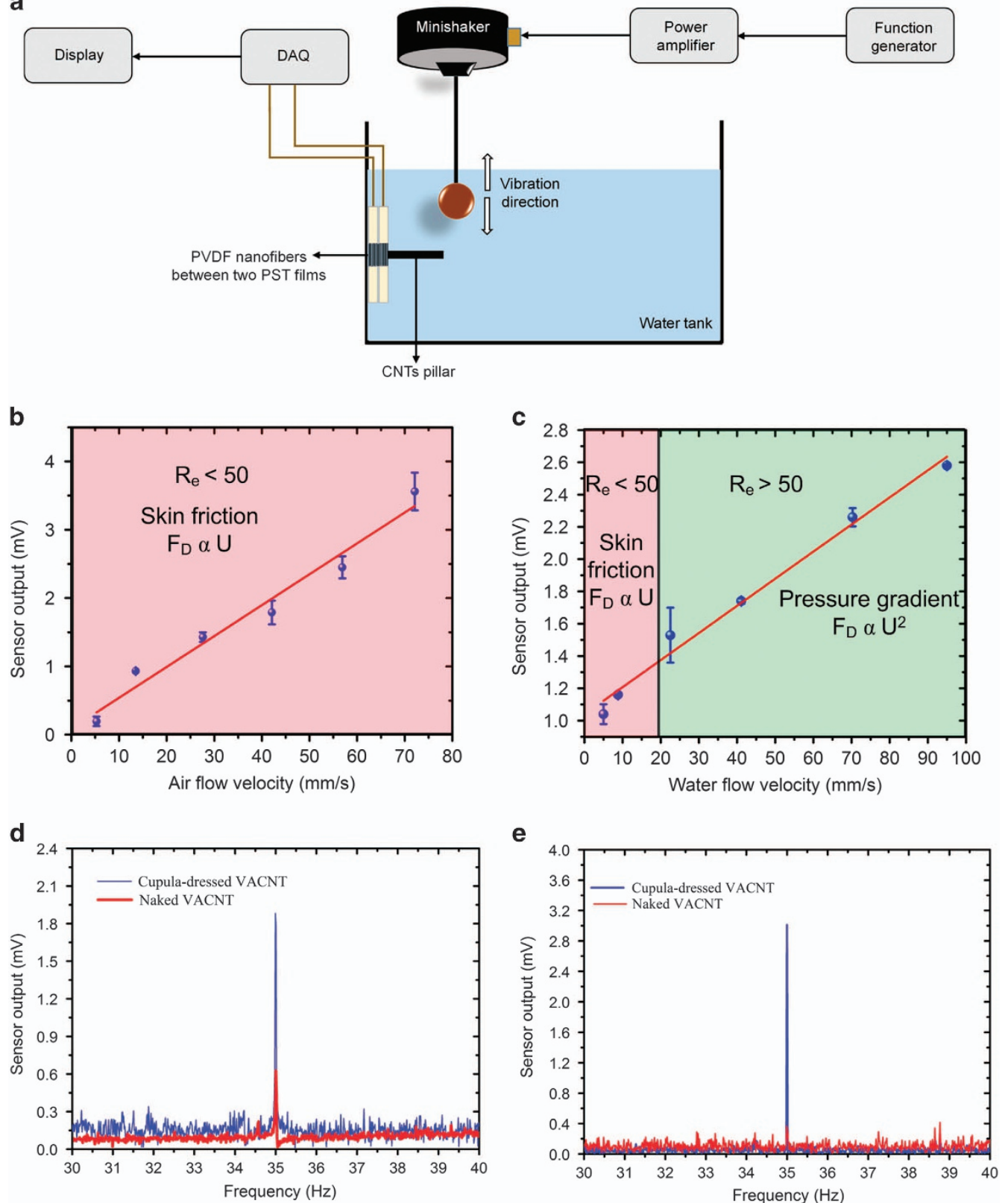

Figure 4 Flow sensing experiments in air and water. (a) Experimental setup using a dipole stimulus to generate oscillatory flow with biological relevance to the lateral-line stimulus. (b) Flow calibration of the naked VACNT sensor in air flow. (c) Flow calibration of the naked VACNT sensor in water flow. Error bars represent the s.d.'s of the sensor outputs for five repeats of the experiment on five sensors. (d, e) Enhancement in sensor output of the HA-Tyr cupuladressed VACNT sensor in comparison to the naked VACNT sensor in air and water flows, respectively.

the pressure gradient determines the drag force. Flow calibration results show that the sensor is capable of sensing low flows with a velocity detection threshold of $5 \mathrm{~mm} \mathrm{~s}^{-1}$ in both air and water. The voltage sensitivity of the sensor for air and water flows is $17.2 \mathrm{mV} /$ $\left(\mathrm{m} \mathrm{s}^{-1}\right)$ and $45.2 \mathrm{mV} /\left(\mathrm{m} \mathrm{s}^{-1}\right)$, respectively.

In another experiment, the naked VACNT sensor and cupuladressed VACNT sensor were simultaneously positioned equidistant from the center of the dipole to determine the enhancement in the sensor output in the presence of the hydrogel cupula. The dipole was vibrated at a frequency of $35 \mathrm{~Hz}$ and an amplitude of $250 \mathrm{mV}_{\mathrm{rms}}$, and the outputs from both sensors were simultaneously recorded. Figures $4 \mathrm{~d}$ and e show the frequency content of the sensor outputs (fast Fourier transform of voltage amplitude output), which clearly shows a peak at $35 \mathrm{~Hz}$ for both sensors. It was also observed that the amplitude of the voltage output of the cupula-dressed sensor increased by 2.5 and 8 times that of the naked VACNT sensor in air and water flows, respectively. A similar enhancement in the sensor output was observed for all frequencies in the range of $1-100 \mathrm{~Hz}$ due to the incorporation of the cupula. The additional data on the repeatability of the experimental sensitivity enhancement is provided in the Supplementary Information (Supplementary Figure S2).

The enhancement in sensitivity due to the presence of the HA-Tyr hydrogel cupula is higher than that reported in our earlier work, which used methacrylic anhydride (MA) modified HA hydrogel (HA-MA) cupula. ${ }^{11}$ This could be attributed to the structural differences between the two types of HA hydrogels. The crosslinked network of hydrogel 
a

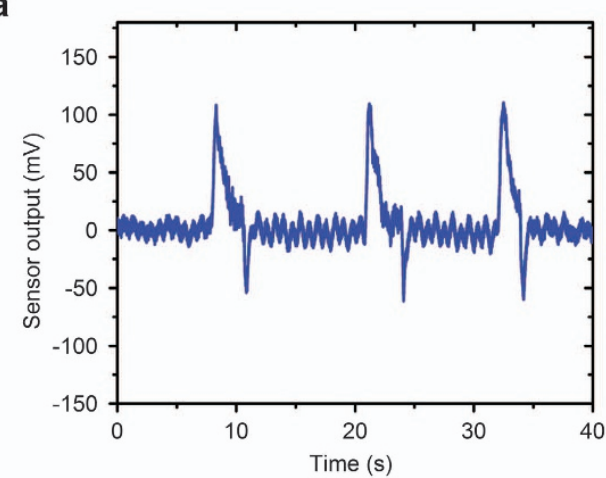

C

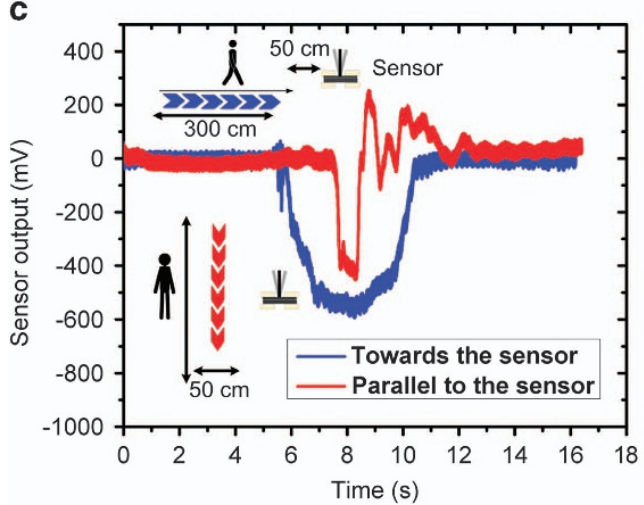

b

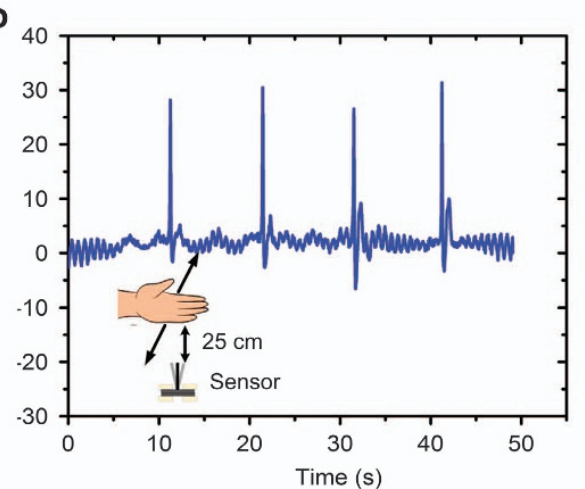

d

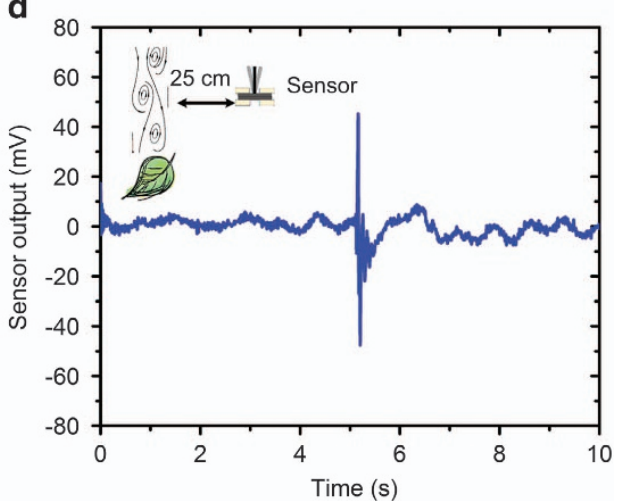

e

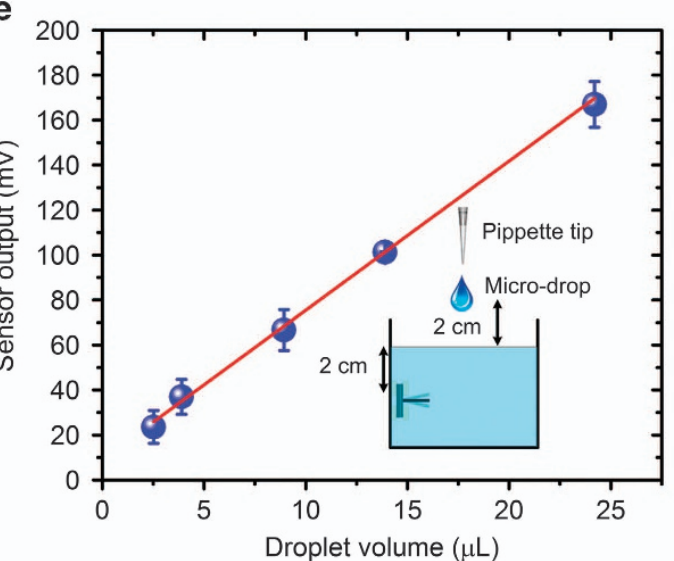

Figure 5 Applications of zero-powered, miniaturized, low-cost, and lightweight PVDF- VACNT sensor for human motion detection and high-precision flow sensing. (a) Response of the sensor to three pulses of air flow velocity of $10 \mathrm{~mm} / \mathrm{s}^{-1}$. (b) Response of the sensor to four sweeps of a human hand at a distance of $25 \mathrm{~cm}$ away from the sensor. (c) Sensor output for a human subject walking parallel (at a distance of $50 \mathrm{~cm}$ from the sensor) and perpendicular (starting from a distance of $3 \mathrm{~m}$ to a distance of $50 \mathrm{~cm}$ from the sensor) to the sensor. (d) Response of the sensor to a leaf dropping at a distance of $10 \mathrm{~cm}$ from the sensor. (e) Underwater sensing experiment demonstrating the high sensitivity of the sensor in detecting water droplets with volumes as low as $2 \mu$ l.

obtained from the two chemical moieties, MA and Tyr, have different pore sizes and swelling ratios, thus resulting in different amounts of water absorbed and retained in their matrices. Higher amounts of water-filled pores in the structure of the HA-Tyr hydrogels results in more fluidic interaction (interaction of the water filled in the pores of hydrogel with the surrounding water) than that in the HA-MA hydrogels, thus leading to an increase in the drag force associated enhancement of the sensitivity in the former. The VACNT bundle served as a good scaffold keeping the soft hydrogel cupula intact even at flow velocities as high as $0.5 \mathrm{~m} \mathrm{~s}^{-1}$.
There are two major factors that lead to an enhancement in the sensitivity of the HA-Tyr hydrogel cupula dressed sensor. The first one is the fluid-structure interaction generated drag force that significantly increases because of the presence of the HA-Tyr cupula. The porous structure of the HA-Tyr hydrogel plays an important role in modifying the pressure transfer from the surrounding flowing water to the VACNT encapsulated hydrogel network, enhancing the drag force experienced by the sensor. The second factor is the cross-sectional area of the structure interacting with the fluid that is increased due to the presence of the HA-Tyr hydrogel cupula as compared to the sensor 
featuring the naked VACNT bundle. The increased cross-sectional area leads to an enhanced drag force that can be estimated using a scaling factor (for flows with low Reynolds numbers) as follows:

$$
\left(\frac{F_{\text {HA-Tyr }}}{F_{\mathrm{VACNT}}}\right)\left(\frac{H_{\text {HA-Tyr }}}{H_{\mathrm{VACNT}}}\right)^{4 / 3}\left(\frac{D_{\text {HA-Tyr }}}{D_{\mathrm{VACNT}}}\right)^{2 / 3}
$$

where $F$ is the drag force, $D$ is the diameter, and $H$ is the height of the structure. Considering the geometrical features of the naked and the cupula dressed sensors Figures $1 \mathrm{~g}$ and $\mathrm{h}$, the drag force due to the presence of cupula was found to increase 2.6 times. This factor closely matches the increase in the output observed in the air flow experiment (Figure 4d). However, this factor is much higher in the experiment conducted in water and that could be attributed to the roles of the material parameters and micro-structural network of the HA-Tyr cupula.

We also conducted several experiments to exemplify the applications of such zero-powered, miniaturized, low-cost, lightweight flow sensors in human motion detection and soft robotics and for sensing flows with ultrahigh precision. Figure 5 a shows the response of the sensor to an extremely low air flow velocity of $10 \mathrm{~mm} \mathrm{~s}^{-1}$ passing the VACNT bundle, which generates a sensor output as high as $100 \mathrm{mV}$ (additional experimental results in Supplementary Figure S4). Video recordings showing the displacement of the naked and hydrogel cupula-dressed VACNT bundles are provided in the Supplementary Information (Supplementary Video 1 and 2, respectively). The sensor produced three distinct peaks in its output in response to the flow velocity that was provided three times at short equidistant intervals. A tiny negative peak was seen due to the recoil of the VACNT bundle after the flow was turned off. We conducted experiments where the sensor detects human motion by sensing the various flows generated by body movements. Figure $5 \mathrm{~b}$ shows the sensor output in response to sweeping motion (four times) of a human hand at a distance of $25 \mathrm{~cm}$ from the sensor (additional experimental results in Supplementary Figure S5). The four distinct peaks generated in the sensor output correspond to the four passes of the hand. Figure $5 \mathrm{c}$ shows the response of the sensor to a human walking parallel (red line) and perpendicular (blue line) to the sensor. In the case of walking parallel to the sensor, the human subject walked in a straight line at a distance of $50 \mathrm{~cm}$ away from the sensor. In the case of walking perpendicular to the sensor, the human subject approached the sensor from a distance of $3 \mathrm{~m}$ and stopped after reaching a distance of $50 \mathrm{~cm}$ from the sensor. When the human subject walked toward the sensor, a single broad peak was observed, and when the human subject walked parallel to the sensor, a voltage dip followed by a peak was observed. This is because in the latter case, as the human subject just passed beyond the sensor, the flow now occurred in the backward direction. Additional experimental results for the detection of human subject motion are presented in Supplementary Figures S6 and S7. Figure 5d shows the output of the sensor in response to a thin leaf that was dropped beside the sensor. Even the extremely low flow generated by a falling leaf resulted in a sensor output of $40 \mathrm{mV}$.

To demonstrate the extremely high flow sensitivity of the sensor in water, we conducted an experiment that involved detection of microdroplets of water. The sensor was immersed $2 \mathrm{~cm}$ deep in water while droplets with volumes varying from 2 to $25 \mu$ l were dropped from a height of $2 \mathrm{~cm}$ from the surface of the water. The sensor was able to detect the flow generated by a $2 \mu$ d droplet and showed a linear increase in the output as the droplet volume increased, as shown in Figure 5e. The error bars represent the s.d.'s for five repeats of the experiment on five sensors. This experimental result demonstrated a

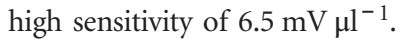

\section{CONCLUSION}

The design of our sensor, although bioinspired from hair cell sensors of fishes, is simplified as compared to the MEMS sensors reported earlier. The HA hydrogel cupula mechanically couples the external flows to the embedded VACNT bundle, thereby enhancing the sensitivity of the sensor. The VACNT bundle not only transfers the flow-generated torque to the PVDF membrane but also acts as a scaffold facilitating the formation of biomimetic hydrogel cupula. Further, the CNT bundle scaffolding hydrogel closely mimics the biological cupula with embedded cupular fibrils, and this biomimetic design offers the benefit of high structural rigidity to the soft cupula. With the CNTs inside the hydrogel matrix, the requirement of the hair cell standing structure proposed in previous MEMS sensor designs is overcome. After swelling, the hydrogel permeates through the CNTs, thereby coupling the flow stimulus with the PVDF nanofibers. The high bendability and elasticity of the CNTs result in a high restoring force that contributes to the faster response time of the sensor. Hence, with our biomimetic sensor, we achieved the function of a hair cell standing structure and cupular fibrils by using a CNT bundle and hydrogel cupula together. This method simplifies the overall sensor design and minimizes the fabrication steps while improving the sensitivity. Unlike biomimetic hair cell sensors developed in the past, this sensor operates with no power supply and achieves a good sensing performance by using the PVDF nanofiber membrane as the sensing material. ${ }^{2-6,8-11,13,21,22}$

\section{CONFLICT OF INTEREST}

The authors declare no conflict of interest.

\section{ACKNOWLEDGEMENTS}

We sincerely thank Dr Tao Kai for the fabrication schematic and amendments in the paper. This research was supported by the National Research Foundation (NRF), Prime Minister's Office, Singapore, under its Campus for Research Excellence and Technological Enterprise (CREATE) program. The Center for Environmental Sensing and Modeling (CENSAM) is an interdisciplinary research group (IRG) of the Singapore MIT Alliance for Research and Technology (SMART) center.

Author contributions: MB conducted the hydrogel preparation, characterization, and drop casting. AGPK conceptualized the idea and conducted the electrospinning and PVDF characterization. MB and AGPK conducted the sensor fabrication, CNT-hydrogel characterization and sensor testing and wrote the manuscript. MB and AGPK contributed equally to this work. MJ and MST conceived and supervised the project. All authors contributed to the discussion of the results and to the critical reading of the manuscript.

\section{PUBLISHER'S NOTE}

Springer Nature remains neutral with regard to jurisdictional claims in published maps and institutional affiliations.

1 Fratzl, P. \& Barth, F. G. Biomaterial systems for mechanosensing and actuation. Nature 462, 442-448 (2009).

2 Rizzi, F., Qualtieri, A., Dattoma, T., Epifani, G. \& De Vittorio, M. Biomimetics of underwater hair cell sensing. Microelectron. Eng. 132, 90-97 (2015).

3 Kottapalli, A. G. P., Asadnia, M., Miao, J. M. \& Triantafyllou, M. S. Touch at a distance sensing: lateral-line inspired MEMS flow sensors. Bioinspir. Biomim 9, 046011 (2014).

4 Fan, Z., Chen, J., Zou, J., Bullen, D., Liu, C. \& Delcomyn, F. Design and fabrication of artificial lateral line flow sensors. J. Micromech. Microeng. 12, 655-661 (2002). 
5 Chen, N., Tucker, C., Engel, J. M., Yang, Y., Pandya, S. \& Liu, C. Design and characterization of artificial haircell sensor for flow sensing with ultrahigh velocity and angular sensitivity. J. Microelectromech. Syst. 16, 999-1014 (2007).

6 Kottapalli, A. G. P., Tan, C. W., Olfatnia, M., Miao, J. M., Barbastathis, G. \& Triantafyllou, M. S. A liquid crystal polymer membrane MEMS sensor for flow rate and flow direction sensing applications. J. Micromech. Microeng 21, 1-11 (2011).

7 Asadnia, M., Kottapalli, A. G. P., Miao, J. M., Warkiani, M. E. \& Triantafyllou, M. S. Artificial fish skin of self-powered micro-electromechanical systems hair cells for sensing hydrodynamic flow phenomena. J. R. Soc. Interface 12, 1-14 (2015).

8 Peleshanko, S., Julian, M. D., Ornatska, M., McConney, M. E., LeMieux, M. C., Chen, N., Tucker, C., Yang, Y., Liu, C., Humphrey, J. A. C. \& Tsukruk, V. V. Hydrogel-encapsulated microfabricated haircells mimicking fish cupula neuromast. Adv. Mater. 19, 2903-2909 (2007).

9 Anderson, K. D., Lu, D., McConney, M. E., Han, T., Reneker, D. H. \& Tsukruk, V. V. Hydrogel microstructures combined with electrospun fibers and photopatterning for shape and modulus control. Polymer 49, 5284-5293 (2008).

10 McConney, M. E., Chen, N., Lu, D., Hu, H. A., Coombs, S., Liu, C. \& Tsukruk, V. V. Biologically inspired design of hydrogel-capped hair sensors for enhanced underwater flow detection. Soft Matter 5, 292-295 (2009).

11 Kottapalli, A. G. P., Bora, M., Asadnia, M., Miao, J. M., Venkatraman, S. S. \& Triantafyllou, M. S. Nanofibril scaffold assisted MEMS artificial hydrogel neuromasts for enhanced sensitivity flow sensing. Sci. Rep. 6, 19336 (2016).

12 Montgomery, J. C., Coombs, S. \& Baker, C. F. The mechanosensory lateral line system of the hypogean form of Astyanax fasciatus. Env. Biol. Fish 62, 87-96 (2001).

13 McConney, M. E., Anderson, K. D., Brott, L. L., Naik, R. R. \& Tsukruk, V. V. Bioinspired material approaches to sensing. Adv. Funct. Mater. 19, 2527-2544 (2009).

14 McHenry, M. J. \& van Netten, S. M. The flexural stiffness of superficial neuromasts in the zebrafish (Danio rerio) lateral line. J. Exp. Biol. 210, 4244-4253 (2007).

15 Bar-Cohen, Y. Introduction to Biomimetics: The Wealth of Inventions in Nature as an Inspiration for Human Innovation In:Biomimetics: Biologically Inspired Technologies (ed.Bar-Cohen Y. Ch. 1), 1-40 (CRC Taylor \& Francis, Florida, USA, 2006).

16 Kochervinskii, V. V. Piezoelectricity in crystallizing ferroelectric polymers: poly (vinylidene fluoride) and its copolymers (a review). Crystallogr. Rep. 48, 649-675 (2003).

17 Sharma, T., Aroom, K., Naik, S., Gill, B. \& Zhang, J. X. J. Flexible Thin-Film PVDF-TrFE Based Pressure Sensor for Smart Catheter Applications. Ann. Biomed. Eng 41, 744-751 (2013).

18 Xin, Y., Guo, C., Qi, X., Tian, H., Li, X., Dai, Q., Wang, S. \& Wang, C. Wearable and unconstrained systems based on PVDF sensors in physiological signals monitoring: A brief review. Ferroelectrics 500, 291-300 (2016).

19 Darr, A. \& Calabro, A. Synthesis and characterization of tyramine-based hyaluronan hydrogels. J. Mater. Sci. Mater. Med 20, 33-44 (2009).
20 Leach, J. B., Bivens, K. A., Patrick, C. W. Jr. \& Schmidt, C. E. Photocrosslinked hyaluronic acid hydrogels: Natural, biodegradable tissue engineering scaffolds. Biotechnol. Bioeng. 85, 578-589 (2003).

21 Dagamseh, A. M. K., Wiegerink, R. J., Lammerink, T. S. J. \& Krijnen, G. J. M. Towards a high-resolution flow camera using artificial hair sensor arrays for flow pattern observations. Bioinsp. Biomim 7, 046009 (2012).

22 Dagamseh, A., Wiegerink, R., Lammerink, T. \& Krijnen, G. Imaging dipole flow sources using an artificial lateral-line system made of biomimetic hair flow sensors. J. R. Soc. Interface. 10, 0162, (2013).

23 Lambricht, L., Berdt, P. D., Vanacker, J., Leprince, J., Diogenes, A., Goldansaz, H., Bouzin, C., Préat, V., Dupont-Gillain, C. \& des Rieux, A. The type and composition of alginate and hyaluronic-based hydrogels influence the viability of stem cells of the apical papilla. Dent. Mater. 30, e349-e361 (2014).

24 Lee, B., Baek, Y., Lee, M., Jeong, D. H., Lee, H. H., Yoon, J. \& Kim, Y. H. A carbon nanotube wall membrane for water treatment. Nat. Commun 6, 7109-7115 (2015).

25 Coombs, S. Nearfield detection of dipole sources by the goldfish (Carassius auratus) and the mottled sculpin (Cottus bairdi). J. Exp. Biol. 190, 109-129 (1994).

26 Yang, Y., Nguyen, N., Chen, N., Lockwood, M., Tucker, C., Hu, H., Bleckmann, H., Liu, C. \& Jones, D. L. Artificial lateral line with biomimetic neuromasts to emulate fish sensing. Bioinsp. Biomim 5, $016001-016009$ (2010).

27 Asadnia, M., Kottapalli, A. G. P., Karavitaki, K. D., Warkiani, M. E., Miao, J. M., Corey, D. P. \& Michael Triantafyllou, M. S. From Biological Cilia to Artificial Flow Sensors: Biomimetic Soft Polymer Nanosensors with High Sensing Performance. Sci. Rep. 6, 32955 (2016).

28 Yang, Y., Chen, J., Engel, J., Pandya, S., Chen, N., Tucker, C., Coombs, S., Jones, D. L. \& Liu, C. Distant touch hydrodynamic imaging with an artificial lateral line. Proc. Natl Acad. Sci. USA 103, 18891-18895 (2006).

(c) (i) This work is licensed under a Creative Commons Attribution 4.0 International License. The images or other third party material in this article are included in the article's Creative Commons license, unless indicated otherwise in the credit line; if the material is not included under the Creative Commons license, users will need to obtain permission from the license holder to reproduce the material. To view a copy of this license, visit http:// creativecommons.org/licenses/by/4.0/

(C) The Author(s) 2017

Supplementary Information accompanies the paper on the NPG Asia Materials website (http://www.nature.com/am) 